\title{
Guest Editorial: Data Management and Analysis in Location-Based Social Networks
}

\author{
Rui Zhang ${ }^{1}$ - Timos Sellis ${ }^{2}$ • Yu Zheng ${ }^{3}$. \\ Mohamed Mokbel ${ }^{4}$
}

Social networks are prevalent on the Internet and become a hot research topic attracting many professionals from a variety of fields. The advances in location-acquisition and mobile communication technologies empower people to use location data with existing online social networks. The dimension of location helps bridge the gap between the physical world and online social networking services. Furthermore, people in an existing social network can expand their social structure with the new interdependency derived from their locations. This Special Issue focuses on the following important research questions in location based social networks. First, how to acquire quality location data which requires matching GPS locations obtained to map locations. Second, how to capture the correlations between the spatial and temporal aspects of the GPS trajectories. Third, how to mine and query the location data effectively and efficiently. Finally, how to deal with location privacy issues in all these tasks. This special issue is

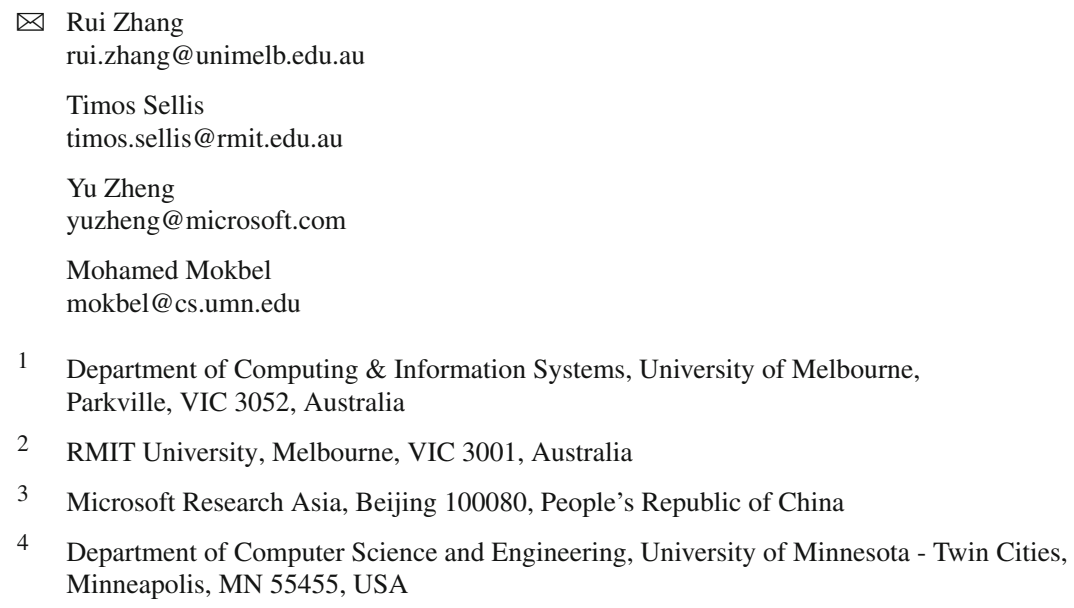


by no means a comprehensive coverage of the above questions, but we have selected five high-quality papers that address some of the challenges. They are summarized below:

- A road network modeling method for map matching on lightweight mobile devices by Peili Wu, Kuien Liu, Kai Zheng, Zhiming Ding, Yuan Tan, studies effective map matching techniques for mobile devices, which have limited storage and computation power.

- LinkNet: Capturing Temporal Dependencies among Spatial Regions by Dhaval Patel, Wynne Hsu, Mong Li Lee, proposes a method to analyze the temporal correlations between events happening at different locations.

- Discovering Pattern-Aware Routes from Trajectories by Ling-Yin Wei, Kai-Ping Chang, Wen-Chih Peng, proposes a framework for discovering driving routes in order to support route planning. The authors approach the problem by mining trajectory patterns and considering user preferences.

- Efficient Route Search on Hierarchical Dynamic Road Networks by Jiajie Xu, Yunjun Gao, Chengfei Liu, Lei Zhao, Zhiming Ding, explores efficient solutions to route planning when there are frequent updates in the traffic networks.

- A Privacy-enhancing Model for Location-based Personalized Recommendations by Jin Huang, Jianzhong Qi, Yabo Xu, Jian Chen, investigates the problem of providing personalized recommendation in the context of location based social networks while protecting the privacy of users.

We would like to thank all the authors for their contribution to this Special Issue.

We would also like to thank the reviewers of this Special Issue for their great efforts in providing reviews. We believe that these selected papers will contribute interesting perspectives to this emerging field.

Rui Zhang, Timos Sellis, Yu Zheng, Mohamed Mokbel

Guest Editors 